\title{
Apendicite aguda não complicada em adultos: tratamento cirúrgico ou clínico?
}

\section{Not complicated acute appendicitis in adults: clinical or surgical treatment?}

\author{
Elcio Shiyoiti Hirano, TCBC-SP1; Bruno Monteiro Tavares Pereira²; Joaquim Murray Bustorff-Silva ${ }^{3}$; Sandro Rizoli4; Bartolomeu \\ Nascimento JR ${ }^{5}$; Gustavo Pereira Fraga, TCBC-SP6
}

INTRODUÇÃO

\begin{abstract}
A apendicite aguda é uma das causas mais frequentes de abdome agudo e pode ser classificada em nãocomplicada e complicada (flegmão e/ou peritonite). Apesar de ter sido originalmente descrita há mais de 125 anos, a etiologia da apendicite aguda continua a ser motivo de debate. Classicamente, a obstrução do apêndice vermiforme por fecalito, corpo estranho, parasitas, hiperplasia linfonodal e tumores, tem sido implicada no desenvolvimento da apendicite aguda. De acordo com esta teoria, a apendicite aguda é considerada uma condição progressiva, que se inicia com o aumento na secreção de muco e a elevação da pressão intraluminal, resultando em estase venosa, compressão arterial e isquemia das paredes do órgão. Com a isquemia da mucosa, o mecanismo protetor de barreira é perdido, levando à invasão bacteriana da parede do apêndice, que por sua vez favorece o infarto e a perfuração do apêndice. Baseado na noção de complicação inevitável, a remoção cirúrgica do apêndice tem sido o tratamento de escolha há mais de um século'. Entretanto, a observação da resolução espontânea de casos de apendicite aguda e os relatos de alguns autores de boa evolução em casos tratados com antibiótico sugerem que nem todos os casos de apendicite aguda enquadram-se na teoria da obstrução mecânica e progressão para apendicite complicada ${ }^{2,3}$. Alguns investigadores sugerem que a apendicite não-complicada e a complicada são duas doenças distintas, com etiologias diversas. Assim como em outros processos intra-abdominais infecciosos como a salpingite, a diverticulite e a enterocolite, que frequentemente são tratadas apenas com antibióticos, a etiologia infecciosa da apendicite aguda é defendida por alguns estudiosos ${ }^{4}$.
\end{abstract}

Apesar da apendicectomia ser a conduta adotada na maioria das instituições, há relatos, desde 1959, do tratamento não operatório com uso de antibioticoterapia em ambas as apresentações clínicas ${ }^{2}$. Nos casos de flegmão apendicular, o tratamento clínico com antibióticos na fase inicial é comumente utilizado por muitos cirurgiões ${ }^{5,6}$.

Nos últimos anos uma série de trabalhos científicos, retrospectivos e prospectivos, tem sido conduzida com o objetivo de comparar o tratamento operatório com o conservador (não operatório) ${ }^{6-10}$. Entretanto, a condução de estudos comparativos nesta área é desafiadora devido aos seguintes fatores: 1) Apendicite aguda é uma doença que possui um espectro de apresentação clínica amplo e vários métodos diagnósticos (clínico, laboratorial, ultrassonográfico, tomográfico e cirúrgico) podem ser utilizados e variar entre os diversos serviços cirúrgicos, o que desafia a classificação diagnóstica de pacientes para inclusão em estudos; 2) A população acometida pela apendicite é heterogênea, incluindo faixas etárias diversas, dificultando a comparação entre pacientes; 3) A taxa de mortalidade geral associada com a apendicite aguda é relativamente baixa, o que torna extremamente difícil avaliar e demonstrar diferenças em mortalidade em trabalhos científicos; 4) A dificuldade em definir "sucesso" ou "superioridade" do tratamento cirúrgico em relação ao conservador, e "equivalência" ou "não inferioridade" da antibioticoterapia em relação à apendicectomia, a fim de permitir uma comparação adequada. A variabilidade do perfil da população estudada, a técnica cirúrgica adotada, os métodos diagnósticos, a escolha e tempo de uso da antibioticoterapia, a periodicidade e os critérios de reavaliação, os critérios de inclusão e exclusão, e os métodos de estudo, são responsáveis pela inconsistência dos

Reunião de Revista TBE-CiTE em 27 de fevereiro de 2012, com a participação dos serviços: Programa de Trauma do Departamento de Cirurgia do Hospital Sunnybrook Health Sciences Centre da Universidade de Toronto, Canadá; Disciplina de Cirurgia do Trauma da Unicamp, Campinas, SP; Disciplina de Cirurgia de Urgência e Trauma do Departamento de Cirurgia e Anatomia da Faculdade de Medicina de Ribeirão Preto da Universidade de São Paulo (USP); Hospital de Base do Distrito Federal, Brasília, DF; Universidade Federal do Amazonas (UFAM), Manaus, AM; e Hospital Universitário Antônio Pedro, Universidade Federal Fluminense, Niterói, RJ.

1. Médico Assistente Doutor da Disciplina de Cirurgia do Trauma do Departamento de Cirurgia da Faculdade de Ciências Médicas da Universidade Estadual de Campinas (Unicamp), Campinas, SP, Brasil; 2. Médico Assistente da Disciplina de Cirurgia do Trauma do Departamento de Cirurgia da Faculdade de Ciências Médicas da Universidade Estadual de Campinas (Unicamp), Campinas, SP, Brasil; 3. Professor Titular da Disciplina de Cirurgia Pediátrica e Chefe do Departamento de Cirurgia da Faculdade de Ciências Médicas da Universidade Estadual de Campinas (Unicamp), Campinas, SP, Brasil; 4. Professor Doutor Associado de Cirurgia e Terapia Intensiva dos Departamentos de Cirurgia e Terapia Intensiva da Universidade de Toronto, Toronto, Canadá; 5. Fellow, Trauma Program, Departamento de Cirurgia da Universidade de Toronto, Toronto, Canadá; 6. Professor Doutor Coordenador da Disciplina de Cirurgia do Trauma do Departamento de Cirurgia da Faculdade de Ciências Médicas da Universidade Estadual de Campinas (Unicamp), Campinas, SP, Brasil. 
resultados tornando difícil a interpretação entre os estudos clinicos $^{6-8}$

As instituições que participaram do TBE-CiTE conduziram uma análise crítica de dois artigos originais e de duas revisões sistemáticas recentes no assunto e geraram recomendações "baseadas nestas evidências" quanto a validade do tratamento não operatório da apendicite aguda não complicada em adulto.

\section{ESTUDO 1}

Estudo clínico randomizado da antibioticoterapia vesus apendicectomia como tratamento inicial da apendicite aguda em pacientes não selecionados ${ }^{9}$.

\section{Justificativa}

O resultado do tratamento não operatorio (antibioticoterapia) da apendicite aguda é incerto. Este estudo foi planejado para avaliar o uso da antibioticoterapia no tratamento da apendicite aguda em pacientes adultos não selecionados.

\section{Pergunta}

É a antibioticoterapia eficaz como primeira escolha para tratar apendicite aguda em adultos?

\section{Achados principais deste estudo}

Estudo prospectivo, que não foi totalmente randomizado, em três hospitais na Suécia que incluiu todos os pacientes adultos (>18 anos) com diagnóstico provável de apendicite (diagnóstico clínico, com ou sem confirmação laboratorial, ultrassonográfico ou tomográfico). Os pacientes foram separados em três grupos: referência (pacientes operados no Hospital Ostra), antibioticoterapia ou cirurgia. A cirurgia podia ser aberta ou laparoscópica enquanto que a antibioticoterapia começava com cefotaxime e metronidazol intravenoso pelas primeiras 24 horas, seguidos de ciprofloxacina e metronidazol oral num total de 10 dias. Os pacientes recebiam um questionário 1 e 12 meses depois e se não respondessem eram contatados por telefone. O estudo tinha dois desfechos principais: eficácia e complicações graves. A definição de eficácia mudou de acordo com o grupo: para antibioticoterapia foi definida como "a não necessidade de operação"; e para o grupo cirúrgico a confirmação que "o diagnóstico de apendicite estava correto ou havia uma doença que requeria operação". Os autores avaliaram vários desfechos secundários, incluindo custo.

Este estudo incluiu 369 pacientes, 202 no grupo de antibióticos e 167 no cirúrgico. Apenas $52 \%$ dos pacientes alocados para antibioticoterapia foram tratados não operatoriamente enquanto que $96 \%$ dos alocados para cirurgia foram operados. Os grupos eram semelhantes em relação ao sexo, idade, dosagem da proteína $C$ reativa, contagem de leucócitos no sangue, temperatura corporal e presença de peritonite local. Apenas 11 dos 119 pacientes tratados com antibiótico tiveram que ser operados, resultando numa eficácia de 90,8\% para antibioticoterapia. Para a apendicectomia a eficácia foi de 89,2\% (diagnóstico correto de apendicite ou outra patologia cirúrgica). Dos 108 pacientes não operados, 15 tiveram apendicite recorrente $(13,9 \%)$ no período de um ano. Um terço das recorrências aconteceram nos primeiros 10 dias e 2/3 entre 3 e 16 meses após a alta hospitalar. Complicações menores foram semelhantes entre os grupos. As complicações maiores foram três vezes mais frequentes em pacientes submetidos ao tratamento cirúrgico $(p<0,05)$. Complicações sem gravidade foram similares nos dois grupos, mas os custos totais foram maiores nos pacientes operados.

\section{Pontos fortes}

O estudo pode ser considerado generalizável já que incluiu todos os pacientes adultos com diagnóstico provável de apendicite aguda; desenho de estudo prospectivo permite uma melhor avaliação de desfechos e coleta de dados; as principais complicações foram avaliadas: reoperação, abscesso, obstrução intestinal, deiscência de sutura, hérnias ou problemas graves da anestesia; relata achados diagnósticos associados com formas complicadas de apendicite. Flegmão e apendicite gangrenosa estão associadas com leucocitose enquanto que a apendicite perfurada está associada com proteína $C$ reativa elevada, leucocitose e aumento da temperatura corporal $(p<0,001)$. Esta informação pode ser usada para ajudar médicos na identificação precoce de doentes que desenvolverão complicações; avalia complicações de acordo com a técnica utilizada. Complicações cirúrgicas foram similares na cirurgia aberta e laparoscópica; avalia desfechos relacionados com o bem estar do paciente. Pacientes tratados com antibiótico tiveram dor abdominal por mais tempo do que o grupo controle.

\section{Limitações}

O estudo não foi completamente randomizado, pois a determinação de incluir o paciente em um determinado grupo do estudo foi feita de acordo com a data de nascimento, e uma vez determinado, os médicos responsáveis poderiam seguir ou não o esquema de randomização do estudo. Devido à impossibilidade de mascarar as intervenções do estudo, isto pode introduzir um viés na randomização de certos pacientes;

Uma taxa alta de troca na randomização. Dos 202 pacientes alocados para antibioticoterapia, praticamente metade (96 pacientes ou 48\%) foram operados, o que diminui a capacidade do estudo em demonstrar um efeito positivo desta intervenção se este efeito realmente existir. Por outro lado, esta troca de randomização pode indicar uma falha nos critérios de inclusão do estudo, ou até mesmo um problema real com a concepção do estudo em si; 
Um índice alto de perda do seguimento de pacientes para avaliar os desfechos do estudo. Metade dos pacientes foi acompanhada por 12 meses. Isto deixa sempre uma interrogação a respeito do resultado das intervenções nos pacientes que não completaram o seguimento:

Inclui tanto apendicites complicadas como não complicadas e não excluiu o flegmão, que é tratado não operatoriamente por muitos cirurgiões;

Não é possível saber com certeza se os grupos são realmente similares. Os dois grupos têm tamanhos diferentes (250 vs. 119), os pacientes operados tinham mais peritonite localizada e difusa; não é possível saber com certeza a gravidade dos pacientes em cada grupo ou quantos de fato tinham apendicite:

Falha em considerar os dois pacientes que tinham um câncer que só foi descoberto por causa da operação;

A população estudada pode não refletir a realidade de países pobres ou daqueles onde o comprometimento com tratamentos prolongados (10 dias) é pequena; nor que 18 anos)

Não avalia a população pediátrica (me-

\section{ESTUDO 2}

Amoxicilina com ácido clavulônico versus apendicectomia para o tratamento da apendicite aguda nao-complicada: um estudo randomizado, controlado, aberto e não-inferior ${ }^{10}$.

\section{Justificativa}

Diversos estudos têm indicado a antibioticoterapia (ATB) como tratamento da apendicite aguda. Entretanto, devido às limitações do ponto de vista metodológico, os estudos não permitem uma conclusão definitiva. Avaliar o uso da amoxacilina + clavulanato versus apendicectomia em pacientes com apendicite aguda não complicada em adultos.

\section{Pergunta}

O uso de antibiótico para o tratamento de apendicite aguda é seguro e tão eficaz quanto a conduta cirúrgica?

\section{Achados principais desse estudo}

A taxa de peritonite até 30 dias fol significantemente maior no grupo tratado com antibióticos. Os pacientes do grupo ATB que foram operados apresentaram maior incidência de complicações pós operatórias no seguimento de um ano.

\section{Pontos fortes}

Desenho do estudo prospectivo, randomizado e controlado, o que é importante para reduzir a possibilidade de viés na formação dos grupos do estudo e tentar distribuir igualmente diferenças que por ventura possam existir entre os pacientes dos dois grupos do estudo. De fato, o grupo da apendicectomia e ATB são bem parecidos em relação às características basais avaliadas; critérios de inclusão bem definidos, diagnóstico feito através de tomografia computadorizada, incluindo apenas os casos de apendicite não complicada baseados em critérios na tomografia; avaliou como desfecho principal do estudo uma complicação comum aos dois tratamentos (peritonite); identificou que a presença de fecalito é um indicador de apendicite complicada ou de insucesso de tratamento com antibióticos; estudo realizado em população definida, no caso, em adultos.

\section{Limitações}

Margem de não inferioridade de 10\%, pode ser considerada elevada;

Comparação do grupo cirúrgico com duas técnicas distintas (videolaparoscópica e aberta) onde a evolução pós operatória pode ser diferente;

Não comparou diretamente o subgrupo dos pacientes alocvados a ATB e que foram operados com o grupo cirúrgico. Foi calculado a taxa de complicações utilizando o número total e não sobre o número de pacientes operados do grupo ATB, o que resulta em um índice menor de morbidade;

Os casos que foram operados no período de 30 dias devido à peritonite do grupo ATB e que no intra operatório não foi confirmado o diagnóstico de apendicite aguda, estes casos não foram considerados na amostra para comparação com grupo cirúrgico;

O antibiótico mencionado na introdução (ertapenem) não foi o antibiótico usado nesse estudo;

Foram utilizados dois aparelhos diferentes de tomografia para o diagnóstico de apendicite;

Não avaliou o custo socio-economico de cada forma de tratamento para o paciente;

Não foram realizadas hemoculturas para melhor avaliação do seguimento do sucesso ou insucesso do tratamento com antibiótico.

\section{ESTUDOS 3 E 4}

O uso exclusivo de antibióticos para o tratamento da apendicite aguda não complicada: uma revisão sistemática e meta-análise ${ }^{7}$.

Apendicectomia versus tratamento com antibiótico para apendicite aguda. (Revisão da Cochrane) ${ }^{8}$.

\section{Justificativa}

Embora o tratamento não operatório já seja uma prática comum em muitos centros nos casos de apendicite complicada por abscesso intraperitoneal, as controvérsias permanecem no que diz respeito à melhor abordagem para a apendicite não complicada. Estudos demonstram resultados inconsistentes e de difícil interpretação. Devido à 
frequência alta da apendicite aguda e certamente grande impacto com a adoção generalizada da antibioticoterapia como primeira escolha no tratamento de sua forma não complicada, a revisão e análise conjunta dos melhores estudos clínicos no tópico são de extrema importância. Duas revisões sistemáticas da literatura pertinente dos últimos 30 anos procuram avaliar as evidências e determinar a utilidade do tratamento com antibiótico em relação à apendicectomia para o tratamento da apendicite aguda.

\section{Perguntas}

Liu e Fogg ${ }^{7}$ : O tratamento não operatório exclusivo, baseado no uso de antibióticos, da apendicite aguda não complicada é efetivo? O tratamento não operatório exclusivo, baseado no uso de antibióticos, da apendicite aguda não complicada é seguro?

Wilms et al.:: O tratamento com antibióticos é tão efetivo quanto a apendicectomia (aberta ou laparoscópica) na cura dentro de duas semanas e sem complicações, avaliadas até um ano, em pacientes com apendicite aguda? Esta revisão considera uma margem de 20\% aceitável para a definição de não inferioridade da antibioticoterapia.

\section{Achados principais desses estudos}

As duas revisões avaliaram quatro estudos clínicos randomizados em comum. Além destes estudos, Liu e Fogg $^{7}$ incluíram um trabalho cientifico retrospectivo próprio e o estudo clínico "quasi-randomizado" e controlado de Hasson et al. ${ }^{9}$, enquanto que Wilms et al. ${ }^{8}$ colegas incluiram o estudo do Vons et al. ${ }^{10}$, discutidos anteriormente neste artigo, totalizando 1.444 pacientes em todos os trabalhos combinados. Apesar de avaliaram praticamente os mesmos estudos, as duas revisões relatam achados distintos e até certo ponto contraditórios.

Liu e Fogg ${ }^{7}$ observaram que o índice de sucesso imediato da antibioticoterapia exclusiva variou de $88,1 \%$ a 100\% nos seis estudos incluídos, com um índice de recorrência tardio de 5,3\% a 14\%, tendo atingido 35\% em um estudo. $O$ índice de complicações do tratamento clínico foi de $0 \%$ em cinco dos seis estudos incluídos e variou de $4,4 \%$ até $34 \%$ nos pacientes submetidos à apendicectomia. Os autores concluiram que: 1 - As evidências sugerem que o tratamento não operatório é eficiente e seguro em pacientes com apendicite não complicada; 2 - Estudos prospectivos, randomizados e controlados são necessários para definir o papel do tratamento não operatório da apendicite não complicada.

Wilms et al. ${ }^{8}$ relataram que $73,4 \%(62,7$ $81,9 \%$; intervalo de confiança [IC] 95\%) e 97,4\% (94,4 98,8\%; IC 95\%) dos pacientes tratados com antibióticos e apendicectomia, respectivamente, foram curados dentro de duas semanas e sem complicações significativas (incluindo recorrência do quadro) até um ano. A margem inferior do IC 95\% de 15,2\% foi menor do que o $20 \%$ aceitável para declarar que a antibioticoterapia não é não-inferior.
Permanência hospitalar foi menor no grupo da apendicetomia. Nehuma diferença significativa foi observada em relação aos dias de licença por motivo de doença, o que foi avaliado em apenas dois estudos. Portanto, os autores concluíram:

Apesar de uma taxa menor de sucesso no grupo antibiótico, o estudo é considerado inconclusivo, devido ao IC que atingiu a margem de $20 \%$ tolerada para declarar que o tratamento é não-inferior;

Devido à qualidade baixa dos estudos, os resultados devem ser interpretados cuidadosamente e conclusões definitivas não podem ser realizadas;

A apendicectomia continua a ser o tratamento padrão para apendicite aguda; a antibioticoterapia exclusiva pode ser usada no contexto de pesquisa ou em situações onde a cirurgia é contra-indicada.

\section{Pontos fortes}

Como toda revisão sistemática e metanálise, um número maior de pacientes combinados geralmente possibilita a realização de uma análise mais definitiva para determinação da eficácia de um tratamento quando comparado com estudos individuais; a revisão realizada por Wilms et al. ${ }^{8}$ segue as recomendações do grupo Cochrane, utilizando uma metodologia científica bem estabelecida e rigorosa, particularmente em relação a avaliação da qualidade dos estudos selecionados e métodos de análise estatística. Esta revisão possui um desfecho principal que permite uma comparação significante entre os estudos e tratamentos. Os autores também avaliaram fatores relacionados com as repercussões para o paciente e os custos das intervenções; após uma avaliação padronizada e estabelecida da qualidade dos estudos selecionados, os autores excluíram o estudo de Hansson et al. ${ }^{9}$, classificado como de qualidade ruim; os dois estudos conduziram uma revisão detalhada da literatura pertinente, com critérios de inclusão dos estudos bem explicitados.

\section{Limitações}

Independente de ser bem conduzida e de alto nível, qualquer revisão sistemática e meta-análise representa a qualidade dos estudos combinados. Na revisão de Wilms et al. ${ }^{8}$ todos os estudos selecionados foram classificados como sendo de qualidade baixa ou moderada;

A revisão de Liu e Fogg ${ }^{7}$ considerou todos os estudos como sendo de qualidade alta. Entretando, os autores utilizaram uma escala de classificação (NewcastleOttawa Quality Assessment Scale for Cohort Studies) indicada para estudos que não são randomizados. Entre os seis estudos incluídos nesta revisão, quatro são estudos randomizados, um "parcialmente randomizado" e apenas um retrospectivo não randomizado;

A maioria dos estudos incluídos nas revisões não relata o uso profilático de antibióticos em pacientes submetidos à cirurgia, que é associado com redução de 
infecção de sítio cirúrgico. Isto pode introduzir um viés em favor do tratamento cirúrgico;

Os estudos não fornecem informação a respeito do número de pacientes inicialmente abordados para entrada nos trabalhos cientificos. Esta informação é importante para se avaliar a opinião dos pacientes em relação ao tratamento estudado. Um número alto de pacientes não concordando em participar num estudo de antibioticoterapia para apendicite sugeriria que este tratamento não é bem aceito;

No estudo de Liu e Fogg ${ }^{7}$, embora o título sugira a realização de uma meta-análise, trata-se na verdade apenas de uma revisão sistemática de estudos comparativos:

Embora quatro dos estudos incluídos sejam definidos como prospectivos randomizados, faltam informações a respeito dos critérios de inclusão, tipo de randomização e outros dados que permitam melhor avaliar o nível de evidência de todos os trabalhos;

Os autores da revisão sistemática da Cochrane aceitaram uma margem de inferioridade de até $20 \%$ na antibioticoterapia em relação à apendicectomia para o desfecho principal do estudo para considerá-la não inferior ${ }^{8}$. Em termos práticos, isto seria o mesmo que aceitar que de cada 5 pacientes tratados com antibióticos, um não seria curado dentro de duas semanas ou teria complicações importantes até um ano de seguimento. Alguns cirurgiões certamente considerariam isto um risco muito elevado para os seus pacientes;

Aparentemente foram incluídos, em alguns desses estudos, pacientes com peritonite localizada ou generalizada, o que dificilmente pode ser classificado com apendicite não complicada, e pode introduzir um viés importante de seleção;

Os métodos de avaliação e diagnóstico da apendicite diferem muito de estudo para estudo tornando muito difícil avaliar o grau de evolução da apendicite nos diversos grupos estudados.

\section{CONCLUSÃO DA TBE-CITE}

As conclusões são baseadas nas publicações discutidas acima e nos artigos que constam nestas revisões.

Existem alguns artigos que sugerem o tratamento clínico com antibióticos para a apendicite aguda, com morbidade e mortalidade semelhantes ou maiores que o tratamento cirúrgico.

A qualidade metodológica dos estudos que comparam a antibioticoterapia com a apendicectomia é o maior fator limitante para obter conclusões mais definitivas no tópico.

Não há evidência de vantagens ou eficácia maior no tratamento de apendicite aguda com antibioticoterapia, seja do ponto de vista clínico-cirúrgico ou sócio-econômico do paciente.
O papel da antibioticoterapia exclusiva no tratamento da apendicite aguda não complicada em adultos precisa ser melhor determinada através de estudos de melhor qualidade.

Não foram avaliados os resultados do tratamento em crianças.

Recomendações da TBE-CiTE sobre "Apencicite aguda não complicada em adultos: tratamento cirúrgico ou clínico?"

1. O tratamento de escolha da apendicite aguda não complicada em adultos continua a ser cirúrgico;

2. O tratamento exclusivo com antibióticos não pode ser recomendado rotineiramente na prática médica atual, devendo apenas ser considerada em pacientes selecionados ou no contexto de estudos clínicos.

\section{REFERENCIAS}

1. McBurney $C$. Experience with early operative interference in cases of disease of the vermiform appendix. NY Med J. 1889;50:67684

2. Coldrey E. Five years of conservative treatment of acute appendicitis. J Int Coll Surg. 1959;32:255-61.

3. Mason RJ. Surgery for appendicitis: is it necessary? Surg Infect. 2008;9(4):481-8.

4. Sisson RG, Ahlvin RC, Harlow MC. Superficial mucosal ulceration and the pathogenesis of acute appendicitis. Am J Surg. 1971;122(3):378-80

5. Vianna AL, Otero PM, Cruz CAT, Carvalho SM, Oliveira PG, Puttini SMB. Tratamento conservador do platrão apendicular. Rev Col Bras Cir. 2003;30(6):442-6.

6. Fitzmaurice GJ, McWilliams B, Hurreiz H, Epanomeritakis E. Antibiotics versus appendectomy in the management of acute appendicitis: a review of the current evidence. Can J Surg. 2011:54(5):307-14.

7. Liu K, Fogg L. Use of antibiotics alone for treatment of uncomplicated acute appendicitis: a systematic review and metaanalysis. Surgery. 2011;150(4):673-83

8. Wilms IM, de Hoog DE, de Visser DC, Janzing HM. Appendectomy versus antibiotic treatment for acute appendicitis. Cochrane Database Syst Rev. 2011;9(11):CD008359.

9. Hansson J, Körner U, Khorram-Manesh A, Solberg A, Lundholm K. Randomized clinical trial of antibiotic therapy versus appendicectomy as primary treatment of acute appendicitis in unselected patients. Br J Surg. 2009;96(5):473-81. Erratum in: Br J Surg. 2009:96(7):830.

10. Vons C, Barry C, Maitre S, Pautrat K, Leconte M, Costaglioli B, et al. Amoxicillin plus clavulanic acid versus appendicectomy for treatment of acute uncomplicated appendicitis: an open-label non-inferiority, randomised controlled trial. Lancet. 2011;377(9777):1573-9

\section{Declaração:}

As recomendações e conclusões deste artigo representam a opinão dos participantes da reunião de revista TBE-CiTE e não necessariamente a opinião das instituições a que eles pertencem.

Recebido em 28/02/2012

Aceito para publicação em 23/03/2012

Conflito de interesse: nenhum

Fonte de financiamento: nenhuma 
Como citar este artigo:

Hirano ES, Pereira BMT, Bustorff-Silva JM, Rizoli S, Nascimento Júnior $B$, Fraga GP. Apendicite aguda não complicada em adultos: tratamento cirúrgico ou clínico?. Rev Col Bras Cir. [periódico na internet] 2012; 39(2). Disponível em URL: http://www.scielo.br/rcbc
Endereço para correspondência:

Gustavo Pereira Fraga

Rua Alexander Fleming, 181

Cidade Universitária "Prof. Zeferino Vaz" - Barão Geraldo

13.083-970 - Campinas, SP

E-mail: fragagp2008@gmail.com 